\title{
Enhanced Two-Step Method via Relaxed Order of $\alpha$-Satisfactory Degrees for Fuzzy Multiobjective Optimization
}

\author{
Na Wang, ${ }^{1,2}$ Chaofang Hu, ${ }^{3}$ Wuxi Shi, ${ }^{1,2}$ Chunbo Xiu, ${ }^{1,2}$ and Yimei Chen ${ }^{1,2}$ \\ ${ }^{1}$ School of Electrical Engineering and Automation, Tianjin Polytechnic University, Tianjin 300387, China \\ ${ }^{2}$ Key Laboratory of Advanced Electrical Engineering and Energy Technology, Tianjin Polytechnic University, Tianjin 300387, China \\ ${ }^{3}$ School of Electrical Engineering and Automation, Tianjin University, Tianjin 300072, China \\ Correspondence should be addressed to Chaofang $\mathrm{Hu}$; cfhu@tju.edu.cn
}

Received 16 September 2013; Revised 31 December 2013; Accepted 14 January 2014; Published 2 March 2014

Academic Editor: Kim-Hua Tan

Copyright (C) $2014 \mathrm{Na}$ Wang et al. This is an open access article distributed under the Creative Commons Attribution License, which permits unrestricted use, distribution, and reproduction in any medium, provided the original work is properly cited.

\begin{abstract}
An enhanced two-step method via relaxed order of satisfactory degrees for fuzzy multiobjective optimization is proposed in this paper. By introducing the concept of fuzzy numbers and $\alpha$-level set theory, fuzzy parameters are taken as variables, and all the objectives are transformed into fuzzy goals involving three fuzzy relations. The order of $\alpha$-satisfactory degrees which means the objectives with higher priority achieving higher satisfactory degree is applied to model preemptive priority requirement. This strict order constraint is relaxed by priority variable to find the preferred solution satisfying optimization and priority. The original optimization problem is divided into two steps to be solved iteratively. The $\mathrm{M}-\alpha$-Pareto optimality of the solution is ensured, and the satisfactory solution can be acquired by regulating the slack parameter $\Delta \delta$ or changing $\alpha$. The numerical examples demonstrate the power of the proposed method.
\end{abstract}

\section{Introduction}

In recent years, Multiobjective Optimization (MOO) problem has become more and more obvious and important in production and economy, where multiple objectives are conflicting, noncommensurable, and imprecise. Many researchers are interested in its study and development [14]. In the real world, MOO problem often takes place in uncertain environment $[5,6]$. In the early works, research on randomness is devoted to uncertainty $[7,8]$. However, there is a qualitatively different type of imprecision which cannot be equated with probability. Therefore, it may lead to a fuzzy understanding for Decision Maker (DM) to the optimization problem $[9,10]$. For example, the goals or parameters of the objectives and constraints are not known precisely in vague environment. Either the perspective goals are given by imprecise DM's judgment or the parameters cannot be determined accurately. Therefore, fuzzy set theory is introduced to represent uncertainty [10-12], where the targets or parameters are modeled using membership functions. Such a MOO problem is called Fuzzy Multiobjective Optimization
(FMOO) problem $[13,14]$. Its research includes different types of FMOO and different preferences [15].

In MOO problem, the final solution is usually dependent upon DM's preference which refers to the opinion of DM concerning points in criterion space. According to the way that DM articulates or incorporates, preference is categorized into prior, posterior, and progressive articulation. Wherein, importance is the commonly prior articulation of preference. In real life, especially for FMOO problem, importance has become the most interesting preference and has attracted many researchers $[16,17]$. It represents the opinion of DM for different objectives and is given by DM in advance. In order to get the preferred result, analyzer needs to model the importance preference mathematically. Generally, weighted method is a traditional strategy about importance, where a scalar criterion is formulated based on $p$-norm $(1 \leq p \leq \infty)$ [18]. As a special case of importance, preemptive priority requirement is used frequently in practice. It means that all the objectives are classified into the different levels according to DM's requirement, and then they are optimized step by 
step. Being the most conventional strategy, the lexicographic method requires that multiple subproblems consisting of different objectives are solved in lexicographic order $[19,20]$. However, this may lead to heavy computation burden. Even degenerative optimization may happen when some objectives with higher priority have obtained the optimum result but the remained ones are not optimized since the solution keeps identical. Thus, Chen and Tsai [21] propose the principle that the objectives with higher priority should have higher satisfactory degree to model the preemptive priority by means of fuzzy theory. Although the multistep optimization procedure is avoided, the satisfactory even feasible solution may not exist because the priority order constraints are too strict. In order to guarantee the feasibility of optimization, Aköz and Petrovic [22] use fuzzy binary relations to represent imprecise priority; however, they only consider the inequality fuzzy relations. In addition, $\mathrm{Hu}$ and $\mathrm{Li}$ [23] present enhanced interactive satisfying optimization method via goal programming, and $\mathrm{Li}$ et al. [24] also design generalized varying-domain optimization approach. Nevertheless, the nonlinearity of the original problem is strengthened, and the more optimization variables are introduced in their methods. This increases computation burden. Therefore, $\mathrm{Li}$ and $\mathrm{Hu}$ [25] propose a two-step satisfactory method for FMOO to acquire the satisfactory solution.

Usually, FMOO problem includes two cases: MOO with fuzzy goals and MOO with fuzzy parameters. Although the original research is resulted from fuzzy goals [10], actually, more and more FMOO problems are mainly oriented from fuzzy parameters. Fuzzy parameters of the constraints will influence the feasible field, and fuzzy parameters of the objectives will decide the optimization result. So they produce more complicated optimization problem than MOO with fuzzy goals. This requires DM to participate in the optimization procedure to provide more information, which brings about more difficulty to obtain the preferred solution. Therefore, more researchers focus on progressive articulation of preference in $\mathrm{MOO}$ with fuzzy parameters such that different interactive methods $[14,26]$ are studied. Nevertheless, finding the preferred solution becomes more difficult when priority preference is required. The above early works $[19,25]$ about priority only adapt to MOO with fuzzy goals, and they are not applied directly to fuzzy parameters. For example, in [25], we have proposed the two-step satisfactory method to handle preemptive priority requirement using relaxed priority constraints. But it only considers the case that the goals are vague. The uncertain objective functions and constraints (i.e., feasible field) that resulted from fuzzy parameters are not involved. This leads to that the previous method cannot be used to solve the MOO model with fuzzy parameters. Moreover, the optimality of solution is not guaranteed for the approach in [25], where there may exist the weak M-Pareto optimal solution. This probably leads to the unreasonable optimization result.

In this paper, the MOO problem with fuzzy parameters and preemptive priority is concerned where fuzzy parameters have possibilistic distributions. The two-step satisfactory method in [25] is introduced and enhanced. Fuzzy parameters are treated as fuzzy numbers. Using the concept of $\alpha$ level set, fuzzy parameters are defined as variables, and all the objectives are regarded as fuzzy goals including three types of fuzzy relations. Then the FMOO problem using $\alpha$-level sets is formulated into an $\alpha$-FMOO problem. For preemptive priority requirement, the relaxed order of $\alpha$ satisfactory degrees denoting that the objective with higher priority achieves the higher $\alpha$-satisfactory degree is applied. Moreover, the original FMOO problem is reformulated into two models, that is, the preliminary optimization model and the priority model. The two models are solved iteratively. The solution of the first model, that is, $\alpha$-maximum overall satisfactory degree, is obtained, and then it is decreased in the second model for balance between optimization and priority. In addition, the value of $\alpha$ also influences the final result and helps to find the optimum value. Thus, the satisfactory solution can be acquired by relaxing $\alpha$-maximum overall satisfactory degree or changing $\alpha$, where the $M-\alpha$-Pareto optimality of the solution is ensured by means of test model. With our method, DM can easily get the preferred result.

In this paper, Section 2 describes MOO problem with fuzzy parameters and preemptive priority requirement. The enhanced two-step method via relaxed order of $\alpha$-satisfactory degrees is proposed in Section 3. Section 4 demonstrates its power by the numerical examples. In Section 5 the conclusion is drawn.

\section{2. $\alpha$-FMOO with Preemptive Priority}

2.1. MOO with Fuzzy Parameters. In practice, the possible values about the parameters of the objectives or constraints are often considered to be imprecise since they often involve the ambiguity of DM's understanding of the real system. These parameters are called fuzzy parameters. Then the MOO problem with fuzzy parameters is described in the following $[14,27]$ :

$$
\begin{array}{ll}
\min & \left(f_{1}\left(\mathbf{x}, \widetilde{\mathbf{a}}_{i}\right), \ldots, f_{k}\left(\mathbf{x}, \widetilde{\mathbf{a}}_{k}\right)\right) \\
\text { s.t. } & \mathbf{x} \in G(\widetilde{\mathbf{b}})=\left\{\mathbf{x} \mid g_{j}\left(\mathbf{x}, \widetilde{\mathbf{b}}_{j}\right) \leq 0, j=1, \ldots, m\right\},
\end{array}
$$

where $f_{i}(\mathbf{x}),(i=1, \ldots, k)$, are multiple objective functions to be minimized and $G \subset R^{n}$ is system constraints. $\widetilde{\mathbf{a}}_{i}=$ $\left(\widetilde{a}_{i 1}, \widetilde{a}_{i 2}, \ldots, \widetilde{a}_{i r_{i}}\right)$ and $\widetilde{\mathbf{b}}_{j}=\left(\widetilde{b}_{j 1}, \widetilde{b}_{j 2}, \ldots, \widetilde{b}_{j s_{j}}\right)$ denote fuzzy parameter vectors. Usually, they are characterized as the fuzzy numbers $[14,27]$. It is reasonable to treat a real fuzzy number as a convex continuous fuzzy subset. There are usually various kinds of membership functions for fuzzy numbers. All of them are continuously mapping, monotonously increasing or decreasing. For example, fuzzy number $\tilde{c}$ is shown in Figure 1. 
Its membership function is written as

$$
\mu_{\widetilde{c}}(c)= \begin{cases}1-\frac{c_{2}-c}{c_{2}-c_{1}}, & c_{1} \leq c \leq c_{2}, \\ 1 & c_{2} \leq c \leq c_{3}, \\ 1-\frac{c-c_{3}}{c_{4}-c_{3}}, & c_{3} \leq c \leq c_{4}, \\ 0, & \text { otherwise. }\end{cases}
$$

Then $\alpha$-level set of fuzzy parameter $(\widetilde{\mathbf{a}}, \widetilde{\mathbf{b}})$ is defined as

$$
(\widetilde{\mathbf{a}}, \widetilde{\mathbf{b}})_{\alpha}=\left\{\begin{array}{l|l}
(\mathbf{a}, \mathbf{b}) \mid \begin{array}{c}
\mu_{\widetilde{a}_{i d}}\left(a_{i d}\right) \geq \alpha, \mu_{\widetilde{b}_{j e}}\left(b_{j e}\right) \geq \alpha \\
i=1,2, \ldots, k ; d=1,2, \ldots, r_{i} \\
j=1,2, \ldots, m ; e=1,2, \ldots, s_{j}
\end{array}
\end{array}\right.
$$

where $\mathbf{a}=\left(\mathbf{a}_{1}, \mathbf{a}_{2}, \ldots, \mathbf{a}_{k}\right)$ and $\mathbf{b}=\left(\mathbf{b}_{1}, \mathbf{b}_{2}, \ldots, \mathbf{b}_{m}\right)$ are taken as the decision variables about $\alpha$.

Since fuzzy parameters will bring about or increase the imprecise nature of DM's judgment about the goals of the objectives, they will result in the fuzzy goals under an $\alpha$ level set. Therefore, DM can give their implicit targets to the objectives. The fuzzy decision is presented as $[10,20,26]$

$$
\begin{array}{ll}
\text { find } & (\mathbf{x}, \mathbf{a}) \\
\text { such that } & f_{i}\left(\mathbf{x}, \mathbf{a}_{i}\right) \longrightarrow f_{i}^{*}, \quad i=1, \ldots, k \\
\text { s.t. } & \mathbf{x} \in G(\mathbf{b})=\left\{\mathbf{x} \mid g_{j}\left(\mathbf{x}, \mathbf{b}_{j}\right) \leq 0, j=1, \ldots, m\right\} \\
& (\mathbf{a}, \mathbf{b}) \in(\widetilde{\mathbf{a}}, \widetilde{\mathbf{b}})_{\alpha},
\end{array}
$$

where $f_{i}^{*}$ is the perspective goal value for the fuzzy objective function $f_{i}\left(\mathbf{x}, \widetilde{\mathbf{a}}_{i}\right)$ and " $\rightarrow$ " denotes three types of fuzzy relations, that is, " $\leq$ " (fuzzy minimization), " $\geq$ " (fuzzy max-

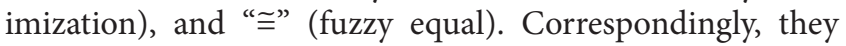
mean that the $i$ th fuzzy objective is approximately less than or equal to $f_{i}^{*}$, approximately more than or equal to $f_{i}^{*}$, and in the vicinity of $f_{i}^{*}$.

In this paper, the conventional linear triangle-like membership functions are adopted to define fuzzy goals of the objective functions with different fuzzy relations. For $(\mathbf{a}, \mathbf{b}) \in$ $(\widetilde{\mathbf{a}}, \widetilde{\mathbf{b}})_{\alpha}$, the membership function under $\alpha$-level set is called $\alpha$-membership function, and its value is called also $\alpha$ satisfactory degree of the fuzzy objective.

For fuzzy minimization " $\leq$ ", the tolerant interval for the fuzzy objective $f_{i}\left(\mathbf{x}, \widetilde{\mathbf{a}}_{i}\right)$ is regarded as $\left(f_{i}^{*}, f_{i}^{\max }\right) . f_{i}^{\max }$ is the tolerant limit. Then the $\alpha$-membership function $\mu_{f_{i}}\left(\mathbf{x}, \mathbf{a}_{i}\right)$ is defined as

$$
\begin{aligned}
& \mu_{f_{i}}\left(\mathbf{x}, \mathbf{a}_{i}\right) \\
& \quad= \begin{cases}1, & f_{i}\left(\mathbf{x}, \mathbf{a}_{i}\right) \leq f_{i}^{*}, \\
1-\frac{f_{i}\left(\mathbf{x}, \mathbf{a}_{i}\right)-f_{i}^{*}}{f_{i}^{\max }-f_{i}^{*}}, & f_{i}^{*} \leq f_{i}\left(\mathbf{x}, \mathbf{a}_{i}\right) \leq f_{i}^{\max }, \\
0, & f_{i}\left(\mathbf{x}, \mathbf{a}_{i}\right) \geq f_{i}^{\max } .\end{cases}
\end{aligned}
$$

It is shown in Figure 2.
For fuzzy maximization " $\geq$ ", the tolerant interval is $\left(f_{i}^{\text {min }}, f_{i}^{*}\right)$. Equation (6) is its $\alpha$-membership function. Figure 3 illustrates the graph of this fuzzy relation:

$$
\begin{aligned}
& \mu_{f_{i}}\left(\mathbf{x}, \mathbf{a}_{i}\right) \\
& = \begin{cases}1, & f_{i}\left(\mathbf{x}, \mathbf{a}_{i}\right) \geq f_{i}^{*}, \\
1-\frac{f_{i}^{*}-f_{i}\left(\mathbf{x}, \mathbf{a}_{i}\right)}{f_{i}^{*}-f_{i}^{\min }}, & f_{i}^{\min } \leq f_{i}\left(\mathbf{x}, \mathbf{a}_{i}\right) \leq f_{i}^{*}, \\
0, & f_{i}\left(\mathbf{x}, \mathbf{a}_{i}\right) \leq f_{i}^{\min } .\end{cases}
\end{aligned}
$$

For “ $\cong$ ", $\left(f_{i}^{\min }, f_{i}^{\max }\right)$ is the tolerant interval (see Figure 4 ). The $\alpha$-membership function is

$$
\begin{aligned}
& \mu_{f_{i}}\left(\mathbf{x}, \mathbf{a}_{i}\right) \\
& \quad= \begin{cases}0, & f_{i}\left(\mathbf{x}, \mathbf{a}_{i}\right) \geq f_{i}^{\max }, \\
1-\frac{f_{i}\left(\mathbf{x}, \mathbf{a}_{i}\right)-f_{i}^{*}}{f_{i}^{\max }-f_{i}^{*}}, & f_{i}^{*} \leq f_{i}\left(\mathbf{x}, \mathbf{a}_{i}\right) \leq f_{i}^{\max }, \\
1 & f_{i}\left(\mathbf{x}, \mathbf{a}_{i}\right)=f_{i}^{*}, \\
1-\frac{f_{i}^{*}-f_{i}\left(\mathbf{x}, \mathbf{a}_{i}\right)}{f_{i}^{*}-f_{i}^{\min },} & f_{i}^{\min } \leq f_{i}\left(\mathbf{x}, \mathbf{a}_{i}\right) \leq f_{i}^{*}, \\
0, & f_{i}\left(\mathbf{x}, \mathbf{a}_{i}\right) \leq f_{i}^{\min } .\end{cases}
\end{aligned}
$$

Introducing the $\alpha$-membership functions of fuzzy goals into (4), FMOO problem is reformulated into the following $\alpha$-FMOO problem:

$$
\begin{array}{ll}
\max & \mu_{f_{1}}\left(\mathbf{x}, \mathbf{a}_{1}\right), \mu_{f_{2}}\left(\mathbf{x}, \mathbf{a}_{2}\right), \ldots, \mu_{f_{k}}\left(\mathbf{x}, \mathbf{a}_{k}\right) \\
\text { s.t. } & \mathbf{x} \in G(\mathbf{b})=\left\{\mathbf{x} \mid g_{j}\left(\mathbf{x}, \mathbf{b}_{j}\right) \leq 0, j=1, \ldots, m\right\} \\
& (\mathbf{a}, \mathbf{b}) \in(\widetilde{\mathbf{a}}, \widetilde{\mathbf{b}})_{\alpha} .
\end{array}
$$

For general $\mathrm{MOO}$ or $\mathrm{MOO}$ under $\alpha$-level set, there is usually Pareto or $\alpha$-Pareto optimality of the solution [14, 28]. Correspondingly, the definition of $\mathrm{M}-\alpha$-Pareto optimality for $\alpha$-FMOO problem (8) is given.

Definition 1 (M- $\alpha$-Pareto optimal solution see [27]). A point $\mathbf{x}^{*} \in G\left(\mathbf{b}^{*}\right)$, where $\left(\mathbf{a}^{*}, \mathbf{b}^{*}\right) \in(\widetilde{\mathbf{a}}, \widetilde{\mathbf{b}})_{\alpha}$, is M- $\alpha$-Pareto optimal solution if and only if there does not exit another solution $\mathbf{x} \in G(\mathbf{b})$ and $(\mathbf{a}, \mathbf{b}) \in(\widetilde{\mathbf{a}}, \widetilde{\mathbf{b}})_{\alpha}$, such that $\mu_{f_{i}}\left(\mathbf{x}, \mathbf{a}_{i}\right) \geq \mu_{f_{i}}\left(\mathbf{x}^{*}, \mathbf{a}_{i}\right)$ for all $i,(i=1, \ldots, k)$ and $\mu_{f_{h}}\left(\mathbf{x}, \mathbf{a}_{i}\right)>\mu_{f_{h}}\left(\mathbf{x}^{*}, \mathbf{a}_{i}\right)$ for at least one $h, h \in\{1, \ldots, k\}$.

Similarly, we can define the concept of Weak M- $\alpha$-Pareto optimal solution.

Definition 2 (weak M- $\alpha$-Pareto optimal solution). A point, $\mathbf{x}^{*} \in \mathrm{G}\left(\mathbf{b}^{*}\right)$, where $\left(\mathbf{a}^{*}, \mathbf{b}^{*}\right) \in(\widetilde{\mathbf{a}}, \widetilde{\mathbf{b}})_{\alpha}$, is weak M- $\alpha$-Pareto optimal solution if and only if there does not exit another solution $\mathbf{x} \in \mathrm{G}(\mathbf{b})$ and $(\mathbf{a}, \mathbf{b}) \in(\widetilde{\mathbf{a}}, \widetilde{\mathbf{b}})_{\alpha}$, such that $\mu_{f_{i}}\left(\mathbf{x}, \mathbf{a}_{i}\right)>$ $\mu_{f_{i}}\left(\mathbf{x}^{*}, \mathbf{a}_{i}\right)$ for all $i,(i=1, \ldots, k)$.

2.2. Preemptive Priority. In real MOO problem, preemptive priority requirement is generally described using linguistic form. For example, suppose the priority of the objective $f_{s}\left(\mathbf{x}, \widetilde{\mathbf{a}}_{s}\right)$ is higher than that of $f_{s^{\prime}}\left(\mathbf{x}, \widetilde{\mathbf{a}}_{s^{\prime}}\right), \quad\left(s, s^{\prime} \in\right.$ 


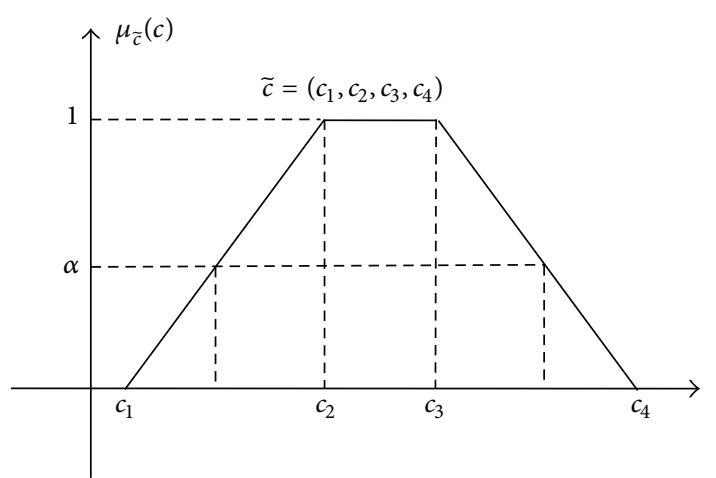

Figure 1: Fuzzy number $\widetilde{c}$.

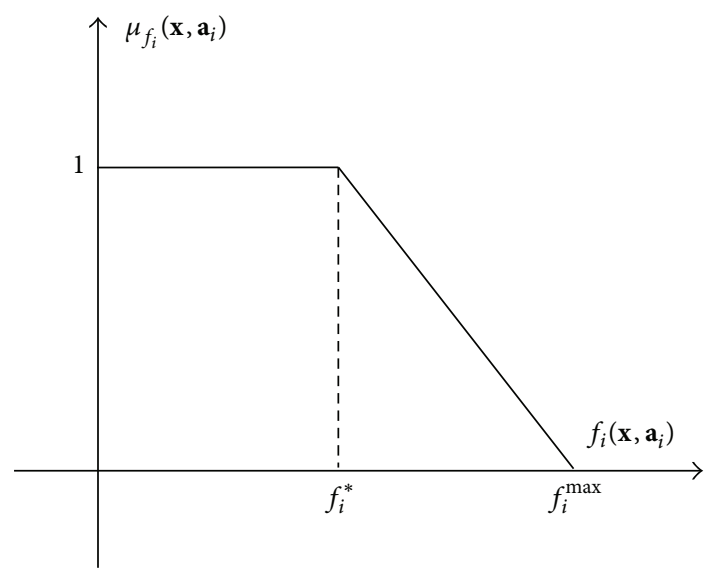

Figure 2: $\alpha$-Membership function $\mu_{f_{i}}\left(\mathbf{x}, \mathbf{a}_{i}\right)$ for fuzzy relation " $\widetilde{\leq}$.

$\left.\{1, \ldots, k\}, s \neq s^{\prime}\right)$. That is, $f_{s}\left(\mathbf{x}, \widetilde{\mathbf{a}}_{s}\right)$ should be optimized before $f_{s^{\prime}}\left(\mathbf{x}, \widetilde{\mathbf{a}}_{s^{\prime}}\right)$. Thus, we can see that preemptive priority has stricter requirement than common importance. It usually requires that all the objectives must be optimized in sequence according to their importance. This means that the objectives with the highest priority must be satisfied firstly, and then the other objectives having the lower priority are considered based on the obtained results. Therefore, all the objectives need to be grouped according to their priority, where there will be different levels including one or several objectives. Consequently, the $\alpha$-FMOO problem with preemptive priority can be formulated as follows [25]:

$$
\begin{array}{ll}
\max \quad[ & P_{1}\left(\mu_{f_{1}^{1}}\left(\mathbf{x}, \mathbf{a}_{1}^{1}\right), \ldots, \mu_{f_{l_{1}}^{1}}\left(\mathbf{x}, \mathbf{a}_{l_{1}}^{1}\right)\right), \ldots, \\
& \left.P_{L}\left(\mu_{f_{1}^{L}}\left(\mathbf{x}, \mathbf{a}_{1}^{L}\right), \ldots, \mu_{f_{l_{L}}^{L}}\left(\mathbf{x}, \mathbf{a}_{l_{L}}^{L}\right)\right)\right] \\
\text { s.t. } \quad & \mathbf{x} \in G(\mathbf{b})=\left\{\mathbf{x} \mid g_{j}\left(\mathbf{x}, \mathbf{b}_{j}\right) \leq 0, j=1, \ldots, m\right\} \\
& (\mathbf{a}, \mathbf{b}) \in(\widetilde{\mathbf{a}}, \widetilde{\mathbf{b}})_{\alpha},
\end{array}
$$

where $P_{i},(i=1, \ldots, L)$ is the priority factor and $P_{i}>>$ $P_{i+1}$. This means the objectives belonging to $P_{i}$ have higher priority than those of $P_{i+1} \cdot f_{1}^{i}\left(\mathbf{x}, \widetilde{\mathbf{a}}_{1}^{i}\right), \ldots, f_{l_{i}}^{i}\left(\mathbf{x}, \widetilde{\mathbf{a}}_{l_{i}}^{i}\right)$ represent the objectives belonging to $i$ th priority level.

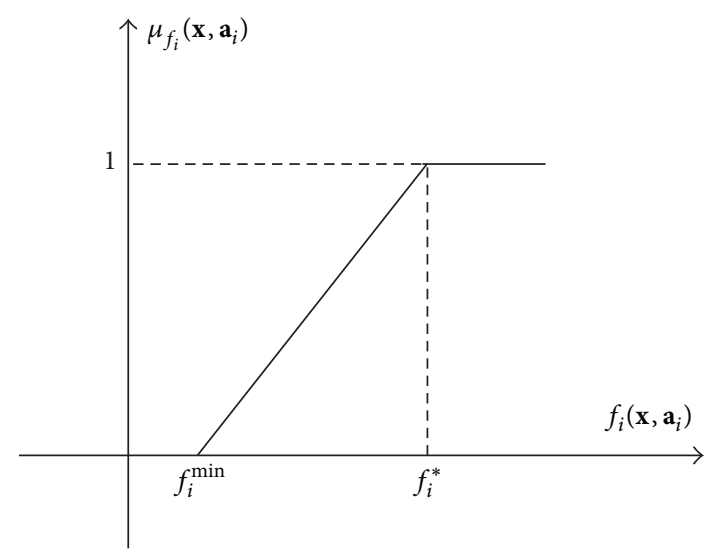

Figure 3: $\alpha$-Membership function $\mu_{f_{i}}\left(\mathbf{x}, \mathbf{a}_{i}\right)$ for fuzzy relation " $\geq$ ".

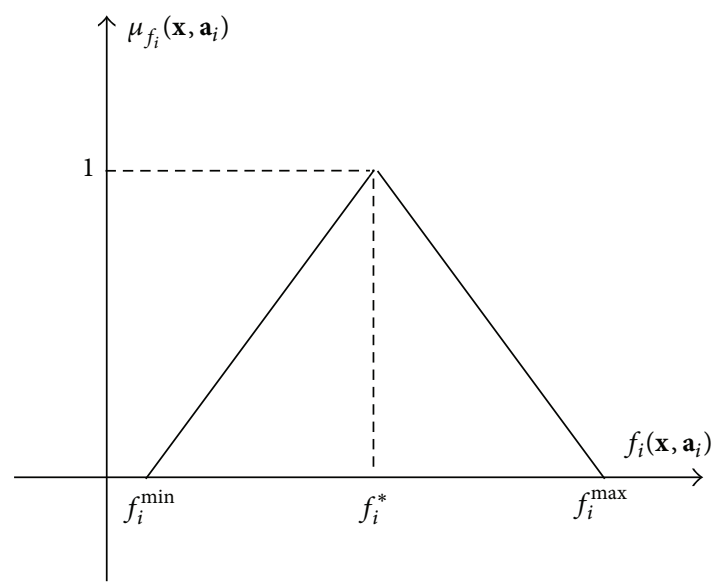

FIGURE 4: $\alpha$-Membership function $\mu_{f_{i}}\left(\mathbf{x}, \mathbf{a}_{i}\right)$ for fuzzy relation “ $\cong$ ”.

\section{Enhanced Two-Step Method via Relaxed Order of $\alpha$-Satisfactory Degrees}

3.1. Relaxed Order of $\alpha$-Satisfactory Degrees. For (9), the traditional strategy is lexicographic optimization [20] whose multistep optimization procedure may result in complex computation even degenerative optimization. According to Chen and Tsai [21], the objective with higher priority should have higher satisfactory degree, by which priority can be presented as the order of satisfactory degrees. It can convert the multistep optimization into a single formulation. This strategy not only adapts to general FMOO but also can be used to $\alpha$-FMOO. For the example about priority in Section 2.2, “ $f_{s}\left(\mathbf{x}, \widetilde{\mathbf{a}}_{s}\right)$ has the higher priority than $f_{s^{\prime}}\left(\mathbf{x}, \widetilde{\mathbf{a}}_{s^{\prime}}\right)$," we can get that the former has the higher $\alpha$-satisfactory degree than the latter. Then the preemptive priority requirement is modeled into the following order of $\alpha$-satisfactory degrees:

$$
\begin{array}{r}
\mu_{f_{s^{\prime}}}\left(\mathbf{x}, \mathbf{a}_{s^{\prime}}\right) \leq \mu_{f_{s}}\left(\mathbf{x}, \mathbf{a}_{s}\right), \quad s, s^{\prime} \in\{1, \ldots, k\}, \\
s \neq s^{\prime}, \quad(\mathbf{a}, \mathbf{b}) \in(\widetilde{\mathbf{a}}, \widetilde{\mathbf{b}})_{\alpha} .
\end{array}
$$


However, the order (10) is too strict. If it is taken as the constraint, the satisfactory even feasible solution maybe cannot be obtained. In addition, the priority difference between the objectives cannot be quantified from (10). This means that the priority requirement may not be reflected accurately when the $\alpha$-satisfactory degrees are the same. Thus, the new priority formulation in [25], " $\mu_{f_{s^{\prime}}}(\mathbf{x})-\mu_{f_{s}}(\mathbf{x}) \leq \gamma$," is adopted, where the priority variable $\gamma,(-1 \leq \gamma \leq 1)$ is introduced to relax strict comparison between the satisfactory degrees. For MOO with fuzzy parameters, this formulation is improved to relax the order of $\alpha$-satisfactory degrees. Then the enhanced preemptive priority is reformulated as follows:

$$
\begin{array}{r}
\mu_{f_{s^{\prime}}}\left(\mathbf{x}, \mathbf{a}_{s^{\prime}}\right)-\mu_{f_{s}}\left(\mathbf{x}, \mathbf{a}_{s}\right) \leq \gamma, \quad s, s^{\prime} \in\{1, \ldots, k\}, \\
s \neq s^{\prime}, \quad(\mathbf{a}, \mathbf{b}) \in(\widetilde{\mathbf{a}}, \widetilde{\mathbf{b}})_{\alpha} .
\end{array}
$$

When $\gamma \leq 0$, (11) denotes that the relaxed order of $\alpha$-satisfactory degrees conforms to the basic preemptive priority order. It is noted that $\gamma=0$ is a special case where priority of all the objectives will be the same. On the contrary, the priority requirement will be violated if $\gamma>0$. By (11), priority difference can be quantified.

\subsection{Enhanced Two-Step Method}

3.2.1. The First Step. In MOO problem, optimization and priority requirements need to be balanced. Therefore, the two-step satisfactory method [25] is introduced. However, fuzzy parameters and three types of fuzzy relations are considered in this paper. This is significantly different from [25] which only focuses on fuzzy goals with the relation of fuzzy minimization. Therefore, the original two-step method needs a great improvement. In this paper, by means of $\alpha$-level set of fuzzy parameters (3) and the relaxed order of $\alpha$-satisfactory degrees (11), $\alpha$-FMOO problem with preemptive priority (9) is divided into two models which consist of the preliminary optimization model and the priority model. They will be solved iteratively.

The purpose of the preliminary optimization model is to optimize all the objectives simultaneously as much as possible regardless of priority. Thus, the first step is to construct and solve the preliminary optimization model. Based on max-min decision, the preliminary optimization model is presented in the following:

$$
\begin{array}{ll}
\max & \lambda \\
\text { s.t. } & \mu_{f_{i}}\left(\mathbf{x}, \mathbf{a}_{i}\right) \geq \lambda, \quad i=1, \ldots, k \\
& \mu_{f_{i}}\left(\mathbf{x}, \mathbf{a}_{i}\right) \leq 1 \\
& \mathbf{x} \in G(\mathbf{b})=\left\{\mathbf{x} \mid g_{j}\left(\mathbf{x}, \mathbf{b}_{j}\right) \leq 0, j=1, \ldots, m\right\} \\
& (\mathbf{a}, \mathbf{b}) \in(\widetilde{\mathbf{a}}, \widetilde{\mathbf{b}})_{\alpha},
\end{array}
$$

where $\lambda$ is called $\alpha$-overall satisfactory degree. It is equal to the minimum $\alpha$-satisfactory degree of all the objectives when preference is not considered. According to max-min decision, the optimal solution of (12) $\lambda^{*}$ is the maximum value of $\alpha$-overall satisfactory degree which denotes that all the objectives are optimized fully. Therefore, it is called $\alpha$-maximum overall satisfactory degree, and it will be treated as the given condition of the next step optimization.

3.2.2. The Second Step. On basis of the first step, we have to consider preemptive priority requirement. Consequently, the priority model is constructed to balance optimization and the priority order in the second step. After solving (12), the feasible field of original $\alpha$-FMOO has become very small. In order to acquire more potential solutions satisfying priority requirement, it is necessary to enlarge the decreased feasible field by the following equations:

$$
\mu_{f_{i}}\left(\mathbf{x}, \mathbf{a}_{i}\right) \geq \lambda^{*}-\Delta \delta, \quad i=1, \ldots, k,
$$

where $\Delta \delta(\Delta \delta \geq 0)$ is slack parameter, by which the $\alpha$ maximum overall satisfactory degree $\lambda^{*}$ can be decreased. This means that there will be more solutions if the constraint (13) is relaxed. Then DM can choose the most satisfactory solution from the new field.

For realizing preemptive priority requirement, the relaxed order of $\alpha$-satisfactory degrees (11) is taken as a new constraint. Combining the original system constraints of (4), the priority model is constructed as follows:

$$
\begin{array}{ll}
\min & \gamma \\
\text { s.t. } & \mu_{f_{i}}\left(\mathbf{x}, \mathbf{a}_{i}\right) \geq \lambda^{*}-\Delta \delta, \quad i=1, \ldots, k \\
& \mu_{f_{s^{\prime}}}\left(\mathbf{x}, \mathbf{a}_{s^{\prime}}\right)-\mu_{f_{s}}\left(\mathbf{x}, \mathbf{a}_{s}\right) \leq \gamma, \\
& s, s^{\prime} \in\{1, \ldots, k\}, \quad s \neq s^{\prime} \\
& -1 \leq \gamma \leq 1 \\
& \mathbf{x} \in G(\mathbf{b})=\left\{\mathbf{x} \mid g_{j}\left(\mathbf{x}, \mathbf{b}_{j}\right) \leq 0, j=1, \ldots, m\right\} \\
& (\mathbf{a}, \mathbf{b}) \in(\widetilde{\mathbf{a}}, \widetilde{\mathbf{b}})_{\alpha} .
\end{array}
$$

In (14), the priority variable $\gamma$ is minimized to enlarge priority difference. During solving, the slack parameter $\Delta \delta$ is regulated in order to acquire the satisfactory solution of $\alpha$-FMOO. In addition to $\Delta \delta$, the feasible field can also be increased by decreasing $\alpha$. These ways provide more regulating freedoms than the original two-step approach in [25]. Therefore, the preferred solution can be found by regulating $\Delta \delta$ or changing $\alpha$.

If $\gamma^{*}>0$, the solution does not conform to priority, $\lambda^{*}$ needs to be decreased through increasing $\Delta \delta$ till $\gamma^{*} \leq 0$, and $\mathrm{DM}$ is satisfied, where $\Delta \delta$ is determined by the interaction between $\mathrm{DM}$ and the analyzer. If the result still remains unsatisfactory after regulating $\Delta \delta$, we can decrease $\alpha$.

3.2.3. Maximum Stable Relaxation. From (13), we know that the feasibility is enlarged continually by increasing $\Delta \delta$ such that the smaller $\gamma^{*}$ can be obtained. When $\Delta \delta$ is increased to equal $\Delta \delta^{*}$, (13) will become inactive constraint. This means $\gamma^{*}$ will remain identical. That is, priority difference will not 
change when $\Delta \delta>\Delta \delta^{*}$. Thus, $\Delta \delta^{*}$ is called maximum stable relaxation. $\boldsymbol{\mu}_{f}^{*}=\left\{\mu_{f_{1}}^{*}, \ldots, \mu_{f_{k}}^{*}\right\}$ is the vector of all the $\alpha$ satisfactory degrees about the optimal solution. $\Delta \delta^{*}$ can be acquired by the following algorithm.

Step 1. Let $\Delta \delta=\lambda^{*}$ and assign a value to $\alpha$, and then solve model (14) to obtain the optimal solution $\mathbf{x}^{*}, \boldsymbol{\mu}_{f}^{*}$ and $\gamma^{*}$.

Step 2. Compare the elements of $\boldsymbol{\mu}_{f}^{*}$, find the minimum one as $\mu_{f_{m}}^{*}$, and let $\Delta \delta^{*}=\lambda^{*}-\mu_{f_{m}}^{*}$.

$\Delta \delta^{*}$ is taken as a priori knowledge by which the case of arbitrary enlarging of the feasible field can be avoided.

3.3. $M$ - $\alpha$-Pareto Optimality Test. From Section 3.2, we know that the preliminary optimization model of the first step is max-min decision on $\infty$-norm, and the $\alpha$-maximum overall satisfactory degree in the priority model of the second step is relaxed. It cannot be guaranteed that each objective under preemptive priority requirement is optimized as much as possible finally, although the optimal solution satisfies the priority order. According to Definition 1, the expected satisfactory solution must be $\mathrm{M}-\alpha$-Pareto optimal. Thus, the following $\mathrm{M}-\alpha$-Pareto optimality test model is proposed. By means of the model, the M- $\alpha$-Pareto optimality of the solution can be ensured:

$$
\begin{array}{ll}
\max & \sum_{i}^{k} \varepsilon_{i} \\
\text { s.t. } & \mu_{f_{i}}\left(\mathbf{x}, \mathbf{a}_{i}\right)-\varepsilon_{i}=\mu_{f_{i}}\left(\mathbf{x}^{*}, \mathbf{a}_{i}\right), \quad i=1, \ldots, k \\
& \mu_{f_{s^{\prime}}}\left(\mathbf{x}, \mathbf{a}_{s^{\prime}}\right)-\mu_{f_{s}}\left(\mathbf{x}, \mathbf{a}_{s}\right) \leq \gamma^{*}, \\
& s, s^{\prime} \in\{1, \ldots, k\}, \quad s \neq s^{\prime} \\
& \mathbf{x} \in G(\mathbf{b})=\left\{\mathbf{x} \mid g_{j}\left(\mathbf{x}, \mathbf{b}_{j}\right) \leq 0, j=1, \ldots, m\right\} \\
& (\mathbf{a}, \mathbf{b}) \in(\widetilde{\mathbf{a}}, \widetilde{\mathbf{b}})_{\alpha} \\
& \varepsilon_{i} \geq 0,
\end{array}
$$

where $\varepsilon_{i},(i=1, \ldots, k)$ are the error variables and $\left(\mathbf{x}^{*}, \gamma^{*}\right)$ is the optimization solution of (14). Let $\overline{\mathbf{x}}$ be the optimal solution of (15). The theorem about $M-\alpha$-Pareto optimality test is given.

Theorem 3. If $\varepsilon_{i},(i=1, \ldots, k)$ are zero, then $\mathbf{x}^{*}$ is $M$ - $\alpha$-Pareto optimal. Otherwise, if at least one $\varepsilon_{h}, h \in\{1, \ldots, k\}$ is not zero, $\overline{\mathbf{x}}$ is the $M-\alpha$-Pareto optimal solution.

Proof. If $\mathbf{x}^{*}$ is not $\mathrm{M}-\alpha$-Pareto optimal when all $\varepsilon_{i}$ are zero, then the optimal solution $\overline{\mathbf{x}}$ must be $\mathrm{M}-\alpha$-Pareto optimal. Because $\mu_{f_{i}}\left(\overline{\mathbf{x}}, \mathbf{a}_{i}\right) \geq \mu_{f_{i}}\left(\mathbf{x}^{*}, \mathbf{a}_{i}\right),(i=1, \ldots, k)$ must hold, and $\mu_{f_{h}}\left(\mathbf{x}, \mathbf{a}_{i}\right)>\mu_{f_{h}}\left(\mathbf{x}^{*}, \mathbf{a}_{i}\right)$ for at least one $h, h \in\{1, \ldots, k\}$. This means there must be one nonzero $\varepsilon_{h}$ at least, which is contrary to the fact that all $\varepsilon_{i}$ are zero. So $\mathbf{x}^{*}$ is $\mathrm{M}-\alpha$-Pareto optimal if $\varepsilon_{i},(i=1, \ldots, k)$ are all zero.
If at least one $\varepsilon_{h}$ is not zero, then $\overline{\mathbf{x}}$ must be the M- $\alpha$ Pareto optimal solution. Or else, there will exist an M- $\alpha$ Pareto optimal solution $\widetilde{\mathbf{x}}$, and it must be the optimal solution of (15). This is contrary to the fact that $\overline{\mathbf{x}}$ is the optimal solution. Therefore $\overline{\mathbf{x}}$ must be $\mathrm{M}-\alpha$-Pareto optimal.

3.4. Algorithm. The specific algorithm of enhanced two-step method is summarized as follows.

Step 1 (initialization). Formulate the $\alpha$-level set of fuzzy parameters, and calculate the individual $f_{i}^{\min }$ and $f_{i}^{\max }$ of the objective function $f_{i}\left(\mathbf{x}, \widetilde{\mathbf{a}}_{i}\right),(i=1, \ldots, k)$ under the given constraints, respectively, when $\alpha=0$ and $\alpha=1$.

Step 2. Determine the desirable targets and the tolerances, construct the $\alpha$-membership functions of all the objectives, and ask DM to select the initial value of $\alpha$.

Step 3. Solve the preliminary optimization model (12) of the first step, and get the $\alpha$-maximum overall satisfactory degree $\lambda^{*}$.

Step 4. Formulate and solve the priority model (14) of the second step according to preemptive priority requirement, and test M-Pareto optimality of the solution by (15).

Step 5. Judge: if $\gamma>0$, go to next step. If $\gamma \leq 0$, but the solution is not satisfactory, also go to Step 6. Otherwise stop optimization, and the satisfactory solution is acquired.

Step 6. Relax $\lambda^{*}$ by increasing $\Delta \delta$ if $\Delta \delta \leq \Delta \delta^{*}$, and go back to Step 4 . Or decrease $\alpha$ and go back to Step 3 .

3.5. Analysis of Feasibility. By the following Theorem, it is proven that there must be the feasible solution in our approach.

Theorem 4. For enhanced two-step method consisting of the preliminary optimization model (12) and the priority model (14), there must exist an optimal solution when $G(\widetilde{\mathbf{b}})$ is nonempty.

Proof. Firstly, the preliminary optimization model (12) of the first step must be feasible because $G(\tilde{\mathbf{b}})$ is nonempty. Secondly, for the priority model (14) of the second step, the relaxed order of $\alpha$-satisfactory degrees (11) can be rewritten as $\left(\mu_{f_{s^{\prime}}}\left(\mathbf{x}, \mathbf{a}_{s^{\prime}}\right)-\mu_{f_{s}}\left(\mathbf{x}, \mathbf{a}_{s}\right) \leq \gamma\right)_{i i}, i i=1, \ldots, Z$, where $Z$ is the number of the priority constraints including active constraints and inactive constraints. Then we have

$$
\gamma=\max _{i i}\left[\left(\mu_{f_{s^{\prime}}}\left(\mathbf{x}, \mathbf{a}_{s^{\prime}}\right)-\mu_{f_{s}}\left(\mathbf{x}, \mathbf{a}_{s}\right)\right)_{i i}\right] .
$$

Thus (14) can be reformulated as

$$
\begin{array}{ll}
\min & \max _{i i}\left[\left(\mu_{f_{s^{\prime}}}\left(\mathbf{x}, \mathbf{a}_{s^{\prime}}\right)-\mu_{f_{s}}\left(\mathbf{x}, \mathbf{a}_{s}\right)\right)_{i i}\right] \\
\text { s.t. } & \mu_{f_{i}}\left(\mathbf{x}, \mathbf{a}_{i}\right) \geq \lambda^{*}-\Delta \delta, \quad i=1, \ldots, k \\
& -1 \leq \max _{i i}\left[\left(\mu_{f_{s^{\prime}}}\left(\mathbf{x}, \mathbf{a}_{s^{\prime}}\right)-\mu_{f_{s}}\left(\mathbf{x}, \mathbf{a}_{s}\right)\right)_{i i}\right] \leq 1
\end{array}
$$


TABLE 1: Fuzzy parameters in Example 1.

\begin{tabular}{lc}
\hline$\widetilde{c}$ & $\left(c_{1}, c_{2}, c_{3}, c_{4}\right)$ \\
\hline$\widetilde{a}_{11}$ & $(-2,-1,-1,0)$ \\
$\widetilde{a}_{12}$ & $(2,3,3,4)$ \\
$\tilde{a}_{2}$ & $(-1,0,0,1)$ \\
\hline
\end{tabular}

$$
\begin{aligned}
& \mathbf{x} \in G(\mathbf{b})=\left\{\mathbf{x} \mid g_{j}\left(\mathbf{x}, \mathbf{b}_{j}\right) \leq 0, j=1, \ldots, m\right\} \\
& (\mathbf{a}, \mathbf{b}) \in(\widetilde{\mathbf{a}}, \widetilde{\mathbf{b}})_{\alpha} .
\end{aligned}
$$

Therefore, the priority model (14) is equivalent to (17). Since $\lambda^{*}$ satisfies the constraints of the preliminary optimization model (12), (13) must hold. Thus, there must be optimal solution for (17). That is, the optimal result also exists in the model (14). Then both of the first step and the second step are feasible. So the optimal solution can be obtained by the enhanced two-step method when $G(\widetilde{\mathbf{b}})$ is nonempty.

\section{Numerical Examples}

We demonstrate the effectiveness of the proposed optimization method by the following numerical examples.

Example 1 (see [25]). The example in [25] is considered, where some parameters of the objectives are imprecise:

$$
\begin{array}{ll}
\min & f_{1}\left(\mathbf{x}, \widetilde{\mathbf{a}}_{1}\right)=\widetilde{a}_{11} x_{1}+3 x_{2}+\widetilde{a}_{12} x_{3} \\
\min & f_{2}\left(\mathbf{x}, \widetilde{\mathbf{a}}_{2}\right)=\left(x_{1}-1\right)^{2}+2\left(x_{2}-3\right)^{2}+\widetilde{a}_{2}\left(x_{3}-2\right)^{2} \\
\text { s.t. } & x_{1}+x_{2}+x_{3} \leq 10 \\
& 0 \leq x_{1}, x_{2}, x_{3} \leq 10 .
\end{array}
$$

$\widetilde{\mathbf{a}}=\left(\widetilde{\mathbf{a}}_{1}, \widetilde{\mathbf{a}}_{2}\right)$ is fuzzy parameter vector, where $\widetilde{\mathbf{a}}_{1}=\left(\widetilde{a}_{11}, \widetilde{a}_{12}\right)$ and $\widetilde{\mathbf{a}}_{2}=\left(\widetilde{a}_{2}\right)$. Their distributions are given in Table 1 .

Preemptive priority requirement is as follows:

level 1: $f_{2}\left(\mathbf{x}, \widetilde{\mathbf{a}}_{2}\right)$,

level 2: $f_{1}\left(\mathbf{x}, \widetilde{\mathbf{a}}_{1}\right)$.

Firstly, optimization simulation is implemented for the given priority, and various results with regard to different $\Delta \delta$ and $\alpha$ are presented. Then maximum stable relaxation $\Delta \delta^{*}$ is computed. Finally, sensitivity analysis is considered by changing the priority order to validate the sensitiveness of our approach.

(1) Optimization for Given Priority. On basis of the proposed method, Example 1 is solved step by step.

(A) The membership functions of $\widetilde{\mathbf{a}}$ are formulated by (2) according to Table 1, and the corresponding constraints about $\alpha$-level set are constructed as $\mathbf{a} \in(\widetilde{\mathbf{a}})_{\alpha}$ using (3). The four optimum values of each objective function are computed when $\alpha=0$ and $\alpha=1$. Thus, the aspiration values and the tolerant limits of the two objectives are determined as
$(-20,40)$ and $(-45,103)$, respectively. The corresponding $\alpha$ membership functions are presented in the following

$$
\begin{gathered}
\mu_{f_{1}}\left(\mathbf{x}, \mathbf{a}_{1}\right)=\frac{\left(40-a_{1} x_{1}-3 x_{2}-a_{2} x_{3}\right)}{60}, \\
\mu_{f_{2}}\left(\mathbf{x}, \mathbf{a}_{2}\right)=\frac{\left(103-\left(x_{1}-1\right)^{2}-2\left(x_{2}-3\right)^{2}-a_{3}\left(x_{3}-2\right)^{2}\right)}{148} .
\end{gathered}
$$

(B) The preliminary model of the first step is written in the following expression:

$$
\begin{array}{ll}
\max & \lambda \\
\text { s.t. } & \mu_{f_{i}}\left(\mathbf{x}, \mathbf{a}_{i}\right) \geq \lambda, \quad \mu_{f_{i}}\left(\mathbf{x}, \mathbf{a}_{i}\right) \leq 1, \quad i=1,2 \\
& \mu_{f_{1}}\left(\mathbf{x}, \mathbf{a}_{1}\right)=\frac{\left(40-a_{11} x_{1}-3 x_{2}-a_{12} x_{3}\right)}{60} \\
& \mu_{f_{2}}\left(\mathbf{x}, \mathbf{a}_{2}\right)=\left(103-\left(x_{1}-1\right)^{2}-2\left(x_{2}-3\right)^{2}\right. \\
& \left.-a_{2}\left(x_{3}-2\right)^{2}\right) \times(148)^{-1} \\
& x_{1}+x_{2}+x_{3} \leq 10 \\
& 0 \leq x_{1}, x_{2}, x_{3} \leq 10 \\
& \mathbf{a} \in(\widetilde{\mathbf{a}})_{\alpha} .
\end{array}
$$

(C) According to the given priority, the relaxed order of $\alpha$-satisfactory degrees is constructed:

$$
\mu_{f_{1}}\left(\mathbf{x}, \mathbf{a}_{1}\right)-\mu_{f_{2}}\left(\mathbf{x}, \mathbf{a}_{2}\right) \leq \gamma
$$

Consequently, the second step model is formulated as

$$
\begin{array}{ll}
\min & \gamma \\
\text { s.t. } & \mu_{f_{i}}\left(\mathbf{x}, \mathbf{a}_{i}\right) \geq \lambda^{*}-\Delta \delta, \quad i=1,2 \\
& \mu_{f_{1}}\left(\mathbf{x}, \mathbf{a}_{1}\right)-\mu_{f_{2}}\left(\mathbf{x}, \mathbf{a}_{2}\right) \leq \gamma \\
& \mu_{f_{1}}\left(\mathbf{x}, \mathbf{a}_{1}\right)=\frac{\left(40-a_{11} x_{1}-3 x_{2}-a_{12} x_{3}\right)}{60} \\
& \mu_{f_{2}}\left(\mathbf{x}, \mathbf{a}_{2}\right)=\left(103-\left(x_{1}-1\right)^{2}-2\left(x_{2}-3\right)^{2}\right. \\
& \\
& x_{1}+x_{2}+x_{3} \leq 10 \\
& 0 \leq x_{1}, x_{2}, x_{3} \leq 10 \\
& -1 \leq \gamma \leq 1 \\
& \mathbf{a} \in(\widetilde{\mathbf{a}})_{\alpha} .
\end{array}
$$


TABLE 2: Optimization results of Example 1 for given priority.

\begin{tabular}{cccccccc}
\hline$\alpha$ & $\lambda^{*}$ & $\Delta \delta$ & $\gamma^{*}$ & $f_{1}\left(\mathbf{x}, \widetilde{\mathbf{a}}_{1}\right)$ & $f_{2}\left(\mathbf{x}, \widetilde{\mathbf{a}}_{2}\right)$ & $\mu_{f_{1}}\left(\mathbf{x}, \mathbf{a}_{1}\right)$ & $\mu_{f_{2}}\left(\mathbf{x}, \mathbf{a}_{2}\right)$ \\
\hline \multirow{3}{*}{0.85} & \multirow{2}{*}{0.6477} & 0.2 & -0.2484 & 13.1349 & -0.0284 & 0.4478 & 0.6961 \\
& & 0.3 & -0.3526 & 19.1374 & -0.6460 & 0.3477 & 0.7003 \\
& & 0.4 & -0.4663 & 25.1374 & -2.6687 & 0.2477 & 0.7140 \\
\hline \multirow{3}{*}{0.65} & 0.6544 & 0.2 & -0.2429 & 12.7386 & -0.1968 & 0.4544 & 0.6973 \\
& & 0.3 & -0.3581 & 18.7386 & -2.4384 & 0.3544 & 0.7124 \\
& & 0.4 & -0.4956 & 24.7386 & -8.0003 & 0.2544 & 0.7500 \\
\hline
\end{tabular}

(D) The M-Pareto optimality test model is written in the following

$$
\begin{array}{ll}
\max & \varepsilon_{1}+\varepsilon_{2} \\
\text { s.t. } & \mu_{f_{i}}\left(\mathbf{x}, \mathbf{a}_{\mathbf{i}}\right)-\varepsilon_{i}=\mu_{f_{i}}\left(\mathbf{x}^{*}, \mathbf{a}_{i}\right), \quad i=1,2 \\
& \mu_{f_{1}}\left(\mathbf{x}, \mathbf{a}_{1}\right)-\mu_{f_{2}}\left(\mathbf{x}, \mathbf{a}_{2}\right) \leq \gamma^{*} \\
& \mu_{f_{1}}\left(\mathbf{x}, \mathbf{a}_{1}\right)=\frac{\left(40-a_{11} x_{1}-3 x_{2}-a_{12} x_{3}\right)}{60} \\
& \mu_{f_{2}}\left(\mathbf{x}, \mathbf{a}_{2}\right)=\left(103-\left(x_{1}-1\right)^{2}-2\left(x_{2}-3\right)^{2}\right. \\
& \\
& x_{1}+x_{2}+x_{3} \leq 10 \\
& 0 \leq x_{1}, x_{2}, x_{3} \leq 10 \times(148)^{-1} \\
& \varepsilon_{i} \geq 0 \\
& \mathbf{a} \in(\widetilde{\mathbf{a}})_{\alpha} .
\end{array}
$$

According to the algorithm, the above models are solved iteratively. The corresponding optimization results are given in Table 2.

Comparing the results listed in Table 2, it is known that the values of $\gamma^{*}$ are less than 0 , and the changing of $\gamma^{*}$ conforms to that of $\Delta \delta$. This means that the preemptive priority requirement is reasonable, and all the results satisfy it. Then DM can choose the preferred solution from them according to his requirement.

(2) Computation of Maximum Stable Relaxation $\Delta \delta^{*}$. From Section 3.2.3, we know that the solution will keep unchanged when $\Delta \delta>\Delta \delta^{*}$. Therefore, there must exist maximum stable relaxation $\Delta \delta^{*}$ during solving Example 1. For different $\alpha, \Delta \delta^{*}$ is correspondingly different. For example, when $\alpha=0.85$ and $\Delta \delta=\lambda^{*}=0.6477, \mu_{f}^{*}=(0.1479,0.7165)$ are obtained by solving (14), where $\mu_{f_{1}}^{*}$ is minimum. Therefore, $\Delta \delta^{*}=0.4998$, which means that the result will remain unchanged when $\Delta \delta>0.4998$.

(3) Sensitivity Analysis. For demonstrating the sensitiveness of the proposed method, sensitivity analysis is constructed by changing the original priority order into the new one " $f_{1}\left(\mathbf{x}, \widetilde{\mathbf{a}}_{1}\right)$ is higher than $f_{2}\left(\mathbf{x}, \widetilde{\mathbf{a}}_{2}\right)$." Then the above models are updated using the new priority constraint
“ $\mu_{f_{2}}\left(\mathbf{x}, \mathbf{a}_{2}\right)-\mu_{f_{1}}\left(\mathbf{x}, \mathbf{a}_{1}\right) \leq \gamma$." They are solved iteratively. The corresponding optimization results are presented in Table 3.

From Table 3, it is seen that the order of $\alpha$-satisfactory degrees changes with alternation of priority requirement. That denotes that our method is sensitive to the priority requirement.

Example 2 (see $[26,27])$. In order to verify the effectiveness of our method further, the example with more objectives from $[26,27]$ is used. Its fuzzy parameters consist in the objectives and the constraints, which makes it more complicated than Example 1:

$$
\begin{aligned}
& \min f_{1}\left(\mathbf{x}, \widetilde{\mathbf{a}}_{1}\right)=\left(x_{1}+5\right)^{2}+\widetilde{a}_{11} x_{2}^{2}+2\left(x_{3}-\widetilde{a}_{12}\right)^{2} \\
& \min f_{2}\left(\mathbf{x}, \widetilde{\mathbf{a}}_{2}\right)=\widetilde{a}_{21}\left(x_{1}-45\right)^{2} \\
& +\left(x_{2}+15\right)^{2}+3\left(x_{3}+\tilde{a}_{22}\right)^{2} \\
& \min f_{3}\left(\mathbf{x}, \widetilde{\mathbf{a}}_{3}\right)=\widetilde{a}_{31}\left(x_{1}+20\right)^{2} \\
& +\widetilde{a}_{32}\left(x_{2}-45\right)^{2}+\left(x_{3}+15\right)^{2} \\
& \text { s.t. } g\left(\mathbf{x}, \widetilde{\mathbf{b}}_{1}\right)=\widetilde{b}_{11} x_{1}^{2}+\widetilde{b}_{12} x_{2}^{2}+\widetilde{b}_{13} x_{3}^{2} \leq 100 \\
& 0 \leq x_{1}, x_{2}, x_{3} \leq 10
\end{aligned}
$$

$\widetilde{\mathbf{a}}=\left(\widetilde{\mathbf{a}}_{1}, \widetilde{\mathbf{a}}_{2}, \widetilde{\mathbf{a}}_{3}\right)$ and $\widetilde{\mathbf{b}}_{1}$ are fuzzy parameter vectors, and $\widetilde{\mathbf{a}}_{1}=\left(\widetilde{a}_{11}, \widetilde{a}_{12}\right), \widetilde{\mathbf{a}}_{2}=\left(\widetilde{a}_{21}, \widetilde{a}_{22}\right), \widetilde{\mathbf{a}}_{3}=\left(\widetilde{a}_{31}, \widetilde{a}_{32}\right)$, and $\widetilde{\mathbf{b}}_{1}=$ $\left(\widetilde{b}_{11}, \widetilde{b}_{12}, \widetilde{b}_{13}\right)$. Their characteristics are presented in Table 4 .

Preemptive priority requirement is as follows:

level 1: $f_{1}\left(\mathbf{x}, \widetilde{\mathbf{a}}_{1}\right)$ and $f_{3}\left(\mathbf{x}, \widetilde{\mathbf{a}}_{3}\right)$,

level 2: $f_{2}\left(\mathbf{x}, \widetilde{\mathbf{a}}_{2}\right)$.

(A) Firstly, the fuzzy parameters $\widetilde{\mathbf{a}}$ and $\widetilde{\mathbf{b}}_{\mathbf{1}}$ are formulated into membership functions about $\alpha$-level set by (3). Then the aspiration values and the tolerant limits of the three objectives are determined as $(2989.5,5932.4),(3485,7997.4)$, and $(7142.5,14008)$, respectively, by means of the minimum and maximum values at $\alpha=0$ and $\alpha=1$. Their $\alpha$ membership functions are defined as 
TABLE 3: Optimization results of Example 1 for sensitivity analysis.

\begin{tabular}{|c|c|c|c|c|c|c|}
\hline$\alpha$ & $\Delta \delta$ & $\gamma^{*}$ & $f_{1}\left(\mathbf{x}, \widetilde{\mathbf{a}}_{1}\right)$ & $f_{2}\left(\mathbf{x}, \widetilde{\mathbf{a}}_{2}\right)$ & $\mu_{f_{1}}\left(\mathbf{x}, \mathbf{a}_{1}\right)$ & $\mu_{f_{2}}\left(\mathbf{x}, \mathbf{a}_{2}\right)$ \\
\hline \multirow{3}{*}{0.85} & 0.2 & -0.3224 & -6.2072 & 36.7388 & 0.7701 & 0.4477 \\
\hline & 0.3 & -0.4501 & -7.8693 & 51.5388 & 0.7978 & 0.3477 \\
\hline & 0.4 & -0.5272 & -9.1950 & 66.3388 & 0.8199 & 0.2477 \\
\hline \multirow{3}{*}{0.65} & 0.2 & -0.3333 & -7.2585 & 35.7552 & 0.7876 & 0.4544 \\
\hline & 0.3 & -0.4659 & -9.2166 & 50.5552 & 0.8203 & 0.3544 \\
\hline & 0.4 & -0.5919 & -10.7764 & 65.3552 & 0.8463 & 0.2544 \\
\hline
\end{tabular}

TABle 4: Fuzzy parameters in Example 2.

\begin{tabular}{lccc}
\hline$\widetilde{c}$ & $\left(c_{1}, c_{2}, c_{3}, c_{4}\right)$ & $\widetilde{c}$ & $\left(c_{1}, c_{2}, c_{3}, c_{4}\right)$ \\
\hline$\widetilde{a}_{11}$ & $(3.8,4,4,4.3)$ & $\widetilde{a}_{32}$ & $(4.7,5,5,5.35)$ \\
$\widetilde{a}_{12}$ & $(48.5,50,50,52)$ & $\widetilde{b}_{11}$ & $(0.9,1,1,1.1)$ \\
$\widetilde{a}_{21}$ & $(1.85,2,2,2.2)$ & $\widetilde{b}_{12}$ & $(0.8,1,1,1.2)$ \\
$\widetilde{a}_{22}$ & $(18.2,20,20,22.5)$ & $\widetilde{b}_{13}$ & $(0.85,1,1,1.15)$ \\
$\tilde{a}_{31}$ & $(2.9,3,3,3.15)$ & & \\
\hline
\end{tabular}

$$
\begin{gathered}
\mu_{f_{1}}\left(\mathbf{x}, \mathbf{a}_{1}\right)=\frac{\left(5932.4-\left(x_{1}+5\right)^{2}-a_{11} x_{2}^{2}-2\left(x_{3}-a_{12}\right)^{2}\right)}{2942.9} \\
\mu_{f_{2}}\left(\mathbf{x}, \mathbf{a}_{2}\right)=\left(7997.4-a_{21}\left(x_{1}-45\right)^{2}-\left(x_{2}+15\right)^{2}\right. \\
\left.-3\left(x_{3}+a_{22}\right)^{2}\right) \times(4512.4)^{-1} \\
\mu_{f_{3}}\left(\mathbf{x}, \mathbf{a}_{3}\right)=\left(14008-a_{31}\left(x_{1}+20\right)^{2}-a_{32}\left(x_{2}-45\right)^{2}\right. \\
\left.-\left(x_{3}+15\right)^{2}\right) \times(6865.5)^{-1} .
\end{gathered}
$$

(B) The preliminary model of the first step is

$\max \lambda$

s.t. $\quad \mu_{f_{i}}\left(\mathbf{x}, \mathbf{a}_{i}\right) \geq \lambda, \quad \mu_{f_{i}}\left(\mathbf{x}, \mathbf{a}_{i}\right) \leq 1, \quad i=1,2,3$

$$
\begin{aligned}
\mu_{f_{1}}\left(\mathbf{x}, \mathbf{a}_{1}\right)= & \left(5932.4-\left(x_{1}+5\right)^{2}-a_{11} x_{2}^{2}\right. \\
& \left.-2\left(x_{3}-a_{12}\right)^{2}\right) \times(2942.9)^{-1} \\
\mu_{f_{2}}\left(\mathbf{x}, \mathbf{a}_{2}\right)=( & 7997.4-a_{21}\left(x_{1}-45\right)^{2}-\left(x_{2}+15\right)^{2} \\
& \left.-3\left(x_{3}+a_{22}\right)^{2}\right) \times(4512.4)^{-1} \\
\mu_{f_{3}}\left(\mathbf{x}, \mathbf{a}_{3}\right)=( & 14008-a_{31}\left(x_{1}+20\right)^{2}-a_{32}\left(x_{2}-45\right)^{2} \\
& \left.-\left(x_{3}+15\right)^{2}\right) \times(6865.5)^{-1} \\
b_{11} x_{1}^{2}+b_{12} x_{2}^{2}+ & b_{13} x_{3}^{2} \leq 100 \\
0 \leq x_{1}, x_{2}, x_{3} \leq & 10
\end{aligned}
$$$$
\left(\mathbf{a}, \mathbf{b}_{1}\right) \in\left(\widetilde{\mathbf{a}}, \widetilde{\mathbf{b}}_{1}\right)_{\alpha} \text {. }
$$

(C) For priority requirement, the relaxed formulation is written as

$$
\begin{aligned}
& \mu_{f_{2}}\left(\mathbf{x}, \mathbf{a}_{2}\right)-\mu_{f_{1}}\left(\mathbf{x}, \mathbf{a}_{1}\right) \leq \gamma \\
& \mu_{f_{2}}\left(\mathbf{x}, \mathbf{a}_{2}\right)-\mu_{f_{3}}\left(\mathbf{x}, \mathbf{a}_{3}\right) \leq \gamma .
\end{aligned}
$$

The second step model is formulated as

$$
\begin{array}{ll}
\min & \gamma \\
\text { s.t. } \quad & \mu_{f_{i}}\left(\mathbf{x}, \mathbf{a}_{i}\right) \geq \lambda^{*}-\Delta \delta, \quad i=1,2,3 \\
& \mu_{f_{2}}\left(\mathbf{x}, \mathbf{a}_{2}\right)-\mu_{f_{1}}\left(\mathbf{x}, \mathbf{a}_{1}\right) \leq \gamma \\
& \mu_{f_{2}}\left(\mathbf{x}, \mathbf{a}_{2}\right)-\mu_{f_{3}}\left(\mathbf{x}, \mathbf{a}_{3}\right) \leq \gamma \\
& \mu_{f_{1}}\left(\mathbf{x}, \mathbf{a}_{1}\right)=\left(5932.4-\left(x_{1}+5\right)^{2}-a_{11} x_{2}^{2}\right. \\
& \left.\quad-2\left(x_{3}-a_{12}\right)^{2}\right) \times(2942.9)^{-1} \\
& \mu_{f_{2}}\left(\mathbf{x}, \mathbf{a}_{2}\right)=\left(7997.4-a_{21}\left(x_{1}-45\right)^{2}-\left(x_{2}+15\right)^{2}\right. \\
& \left.\quad-3\left(x_{3}+a_{22}\right)^{2}\right) \times(4512.4)^{-1} \\
& \mu_{f_{3}}\left(\mathbf{x}, \mathbf{a}_{3}\right)=\left(14008-a_{31}\left(x_{1}+20\right)^{2}-a_{32}\left(x_{2}-45\right)^{2}\right. \\
& \left.\quad-\left(x_{3}+15\right)^{2}\right) \times(6865.5)^{-1} \\
& b_{11} x_{1}^{2}+b_{12} x_{2}^{2}+b_{13} x_{3}^{2} \leq 100 \\
0 \leq x_{1}, x_{2}, x_{3} \leq 10 & \\
-1 \leq \gamma \leq 1 & \left(\mathbf{a}, \mathbf{b}_{1}\right) \in\left(\widetilde{\mathbf{a}}, \widetilde{\mathbf{b}}_{1}\right)_{\alpha} .
\end{array}
$$

(D) The corresponding M-Pareto optimality test model is given in the following

$$
\begin{array}{ll}
\max & \varepsilon_{1}+\varepsilon_{2}+\varepsilon_{3} \\
\text { s.t. } & \mu_{f_{i}}\left(\mathbf{x}, \mathbf{a}_{i}\right)-\varepsilon_{i}=\mu_{f_{i}}\left(\mathbf{x}^{*}, \mathbf{a}_{i}\right), \quad i=1,2,3 \\
& \mu_{f_{2}}\left(\mathbf{x}, \mathbf{a}_{2}\right)-\mu_{f_{1}}\left(\mathbf{x}, \mathbf{a}_{1}\right) \leq \gamma^{*} \\
& \mu_{f_{2}}\left(\mathbf{x}, \mathbf{a}_{2}\right)-\mu_{f_{3}}\left(\mathbf{x}, \mathbf{a}_{3}\right) \leq \gamma^{*}
\end{array}
$$


TABle 5: Optimization results of Example 2.

\begin{tabular}{cccccccccc}
\hline$\alpha$ & $\lambda$ & $\Delta \delta$ & $\gamma$ & $f_{1}\left(\mathbf{x}, \widetilde{\mathbf{a}}_{1}\right)$ & $f_{2}\left(\mathbf{x}, \widetilde{\mathbf{a}}_{2}\right)$ & $f_{3}\left(\mathbf{x}, \widetilde{\mathbf{a}}_{3}\right)$ & $\mu_{f_{1}}\left(\mathbf{x}, \mathbf{a}_{1}\right)$ & $\mu_{f_{2}}\left(\mathbf{x}, \mathbf{a}_{2}\right)$ & $\mu_{f_{3}}\left(\mathbf{x}, \mathbf{a}_{3}\right)$ \\
\hline \multirow{3}{*}{0.9} & \multirow{2}{*}{0.5834} & 0.1 & -0.1619 & 4033.2 & 5815.9 & 9577.4 & 0.6453 & 0.4834 & 0.6453 \\
& & 0.2 & -0.3109 & 3889.1 & 6267.2 & 9241.2 & 0.6943 & 0.3834 & 0.6943 \\
& & 0.3 & -0.4457 & 3786.7 & 6718.4 & 9002.2 & 0.7291 & 0.2834 & 0.7291 \\
\hline \multirow{3}{*}{0.8} & 0.5937 & 0.1 & -0.1621 & 4002.4 & 5769.5 & 9505.6 & 0.6558 & 0.4937 & 0.6558 \\
& & 0.2 & -0.3112 & 3857.8 & 6220.8 & 9168.2 & 0.7049 & 0.3937 & 0.7049 \\
& & 0.3 & -0.4462 & 3755 & 6672 & 8928.3 & 0.7399 & 0.2937 & 0.7399 \\
\hline
\end{tabular}

$$
\begin{aligned}
& \mu_{f_{1}}\left(\mathbf{x}, \mathbf{a}_{1}\right)=\left(5932.4-\left(x_{1}+5\right)^{2}-a_{11} x_{2}^{2}\right. \\
&\left.-2\left(x_{3}-a_{12}\right)^{2}\right) \times(2942.9)^{-1} \\
& \mu_{f_{2}}\left(\mathbf{x}, \mathbf{a}_{2}\right)=\left(7997.4-a_{21}\left(x_{1}-45\right)^{2}-\left(x_{2}+15\right)^{2}\right. \\
&\left.-3\left(x_{3}+a_{22}\right)^{2}\right) \times(4512.4)^{-1} \\
& \mu_{f_{3}}\left(\mathbf{x}, \mathbf{a}_{3}\right)=\left(14008-a_{31}\left(x_{1}+20\right)^{2}-a_{32}\left(x_{2}-45\right)^{2}\right. \\
&\left.-\left(x_{3}+15\right)^{2}\right) \times(6865.5)^{-1} \\
& b_{11} x_{1}^{2}+b_{12} x_{2}^{2}+b_{13} x_{3}^{2} \leq 100 \\
& 0 \leq x_{1}, x_{2}, x_{3} \leq 10 \\
& \varepsilon_{i} \geq 0
\end{aligned}
$$$$
\left(\mathbf{a}, \mathbf{b}_{1}\right) \in\left(\widetilde{\mathbf{a}}, \widetilde{\mathbf{b}}_{1}\right)_{\alpha} .
$$

When $\alpha=0.9$ and $\alpha=0.8$, the models in (B), (C), and (D) are solved iteratively. The optimization results are shown in Table 5.

From Table 5, we know that all the results satisfy the preemptive priority requirement. DM can choose the preferred solution from them. For example, when $\alpha=0.8$ and $\Delta \delta=0.2$, the satisfactory degrees are $0.7049,0.3937$, and 0.7049 , and the values of the objectives are 3857.8, 6220.8, and 9168.2. This may be regarded as the most reasonable result.

Remark 5. The priority variable $\gamma^{*}$ will decrease with the increment of $\Delta \delta$. How to change $\Delta \delta$ can be determined according to the real optimization problem. Usually, it is proper to change $\Delta \delta$ about 0.1 every time.

\section{Conclusion}

This paper addresses the problem of FMOO that resulted from fuzzy parameters. Although the approach in [25] can work with preemptive priority requirement, it only focuses on fuzzy goals but not fuzzy parameters. Therefore, the original two-step method is greatly improved for fuzzy parameters and priority. In this work, the concept of fuzzy number and $\alpha$-level set theory are introduced to adapt to fuzzy parameters. Only inequality fuzzy relations are considered in [25]. However, the enhanced method is extended to three relations involving fuzzy minimization, fuzzy maximization, and fuzzy equal. Based on the previous approach [25], the strict preemptive priority structure is modeled by the relaxed order of $\alpha$-satisfactory degrees. Moreover, there is the possibility of existence of the weak M-Pareto optimal solution in the original approach. Nevertheless, the M-Pareto optimality can be guaranteed by this work. The examples show that regulating $\Delta \delta$ and $\alpha$ provides more optimization freedoms than the previous work during solving the two-step models iteratively. Thus, balance between optimization and priority can be realized when fuzzy parameters are involved. However, we still need to note that the results of some FMOO problems may be so insensitive to the change of $\Delta \delta$ or $\alpha$ that it is difficult to seek the satisfactory solution. Therefore, the sensitivity research on regulating $\Delta \delta$ or $\alpha$ will be studied in the future.

\section{Conflict of Interests}

The authors declare that there is no conflict of interests regarding the publication of this paper.

\section{Acknowledgments}

This work was supported by the National Nature Science Foundation of China under Grant nos. 61074064 and 61203302, Nature Science Foundation of Tianjin 12JCZDJC30300 and 11JCYBJC07000, and the Key Laboratory of Advanced Electrical Engineering and Energy Technology, Tianjin Polytechnic University. The authors are grateful to the anonymous reviewers for their helpful comments and constructive suggestions with regard to this paper.

\section{References}

[1] Y. J. Lai and C. L. Hwang, Fuzzy Multiple Objective Decision Making: Methods and Applications, Springer, Berlin, Germany, 1994.

[2] C. T. Bornstein, N. Maculan, M. Pascoal, and L. L. Pinto, "Multiobjective combinatorial optimization problems with a cost and several bottleneck objective functions: an algorithm with reoptimization," Computers \& Operations Research, vol. 39, no. 9, pp. 1969-1976, 2012.

[3] S.-J. Wu, C.-T. Wu, and J.-Y. Chang, "Fuzzy-based selfinteractive multiobjective evolution optimization for reverse engineering of biological networks," IEEE Transactions on Fuzzy Systems, vol. 20, no. 5, pp. 865-882, 2012. 
[4] C. J. Carmona, P. González, M. J. del Jesus, and F. Herrera, "NMEEF-SD: non-dominated multiobjective evolutionary algorithm for extracting fuzzy rules in subgroup discovery," IEEE Transactions on Fuzzy Systems, vol. 18, no. 5, pp. 958-970, 2010.

[5] O. Crespo, J. E. Bergez, and F. Garcia, "Multiobjective optimization subject to uncertainty: application to irrigation strategy management," Computers and Electronics in Agriculture, vol. 74, no. 1, pp. 145-154, 2010.

[6] A. Dietz, A. Aguilar-Lasserre, C. Azzaro-Pantel, L. Pibouleau, and S. Domenech, "A fuzzy multiobjective algorithm for multiproduct batch plant: application to protein production," Computers and Chemical Engineering, vol. 32, no. 1-2, pp. 292306, 2008.

[7] A. Adeyefa and M. Luhandjula, "Multiobjective stochastic linear programming: an overview," American Journal of Operations Research, vol. 1, no. 4, pp. 203-213, 2011.

[8] F. B. Abdelaziz and H. Masri, "A compromise solution for the multiobjective stochastic linear programming under partial uncertainty," European Journal of Operational Research, vol. 202, no. 1, pp. 55-59, 2010.

[9] M. K. Luhandjula and M. J. Rangoaga, "An approach for solving a fuzzy multiobjective programming problem," European Journal of Operational Research, vol. 232, no. 2, pp. 249-255, 2014.

[10] R. E. Bellman and L. A. Zadeh, "Decision-making in a fuzzy environment," Management Science, vol. 17, no. 4, pp. 141-164, 1970.

[11] H.-J. Zimmermann, "Fuzzy programming and linear programming with several objective functions," Fuzzy Sets and Systems, vol. 1 , no. 1, pp. 45-55, 1978.

[12] H. Tanaka, T. Okuda, and K. Asai, "On fuzzy mathematical programming," Journal of Cybernetics, vol. 3, no. 4, pp. 37-46, 1973.

[13] Y. Gao, G. Q. Zhang, J. Ma, and J. Lu, "A $b m \lambda$-cut and goal-programming-based algorithm for fuzzy-linear multipleobjective bilevel optimization," IEEE Transactions on Fuzzy Systems, vol. 18, no. 1, pp. 1-13, 2010.

[14] S. Li and C. Hu, "An interactive satisfying method based on alternative tolerance for multiple objective optimization with fuzzy parameters," IEEE Transactions on Fuzzy Systems, vol. 16, no. 5, pp. 1151-1160, 2008.

[15] R. T. Marler and J. S. Arora, "Survey of multi-objective optimization methods for engineering," Structural and Multidisciplinary Optimization, vol. 26, no. 6, pp. 369-395, 2004.

[16] C.-C. Lin, "A weighted max-min model for fuzzy goal programming," Fuzzy Sets and Systems, vol. 142, no. 3, pp. 407-420, 2004.

[17] S. Li and C. Hu, "Satisfying optimization method based on goal programming for fuzzy multiple objective optimization problem," European Journal of Operational Research, vol. 197, no. 2, pp. 675-684, 2009.

[18] J.-B. Yang, "Minimax reference point approach and its application for multiobjective optimization," European Journal of Operational Research, vol. 126, no. 3, pp. 541-556, 2000.

[19] R. N. Tiwari, S. Dharmar, and J. R. Rao, "Fuzzy goal programming - an additive model," Fuzzy Sets and Systems, vol. 24, no. 1, pp. 27-34, 1987.

[20] B. B. Pal and B. N. Moitra, "A goal programming procedure for solving problems with multiple fuzzy goals using dynamic programming," European Journal of Operational Research, vol. 144, no. 3, pp. 480-491, 2003.
[21] L.-H. Chen and F.-C. Tsai, "Fuzzy goal programming with different importance and priorities," European Journal of Operational Research, vol. 133, no. 3, pp. 548-556, 2001.

[22] O. Aköz and D. Petrovic, "A fuzzy goal programming method with imprecise goal hierarchy," European Journal of Operational Research, vol. 181, no. 3, pp. 1427-1433, 2007.

[23] C. Hu and S. Li, "Enhanced interactive satisfying optimization approach to multiple objective optimization with preemptive priorities," International Journal of Information Technology \& Decision Making, vol. 5, no. 1, pp. 47-63, 2006.

[24] S. Li, Y. Yang, and C. Teng, "Fuzzy goal programming with multiple priorities via generalized varying-domain optimization method," IEEE Transactions on Fuzzy Systems, vol. 12, no. 5, pp. 596-605, 2004.

[25] S. Li and C. Hu, "Two-step interactive satisfactory method for fuzzy multiple objective optimization with preemptive priorities," IEEE Transactions on Fuzzy Systems, vol. 15, no. 3, pp. 417-425, 2007.

[26] C. Mohan and H. T. Nguyen, "Reference direction interactive method for solving multiobjective fuzzy programming problems," European Journal of Operational Research, vol. 107, no. 3, pp. 599-613, 1998.

[27] M. Sakawa and H. Yano, "An interactive fuzzy satisficing method for multiobjective nonlinear programming problems with fuzzy parameters," Fuzzy Sets and Systems, vol. 30, no. 3, pp. 221-238, 1989.

[28] M. Sakawa, H. Yano, and T. Yumine, "An interactive fuzzy satisficing method for multiobjective linear-programming problems and its application," IEEE Transactions on Systems, Man and Cybernetics, vol. 17, no. 4, pp. 654-661, 1987. 


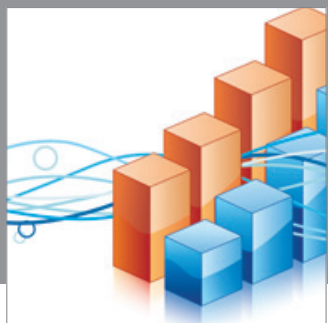

Advances in

Operations Research

mansans

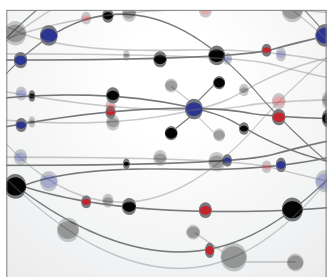

The Scientific World Journal
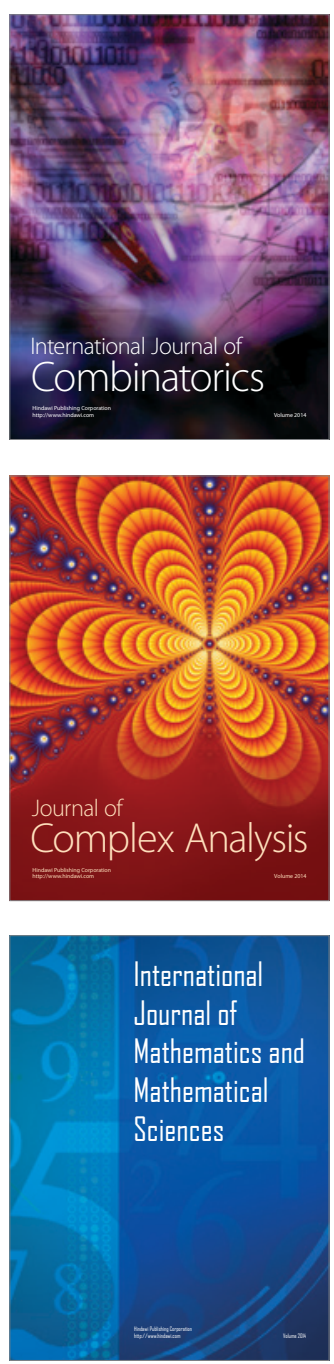
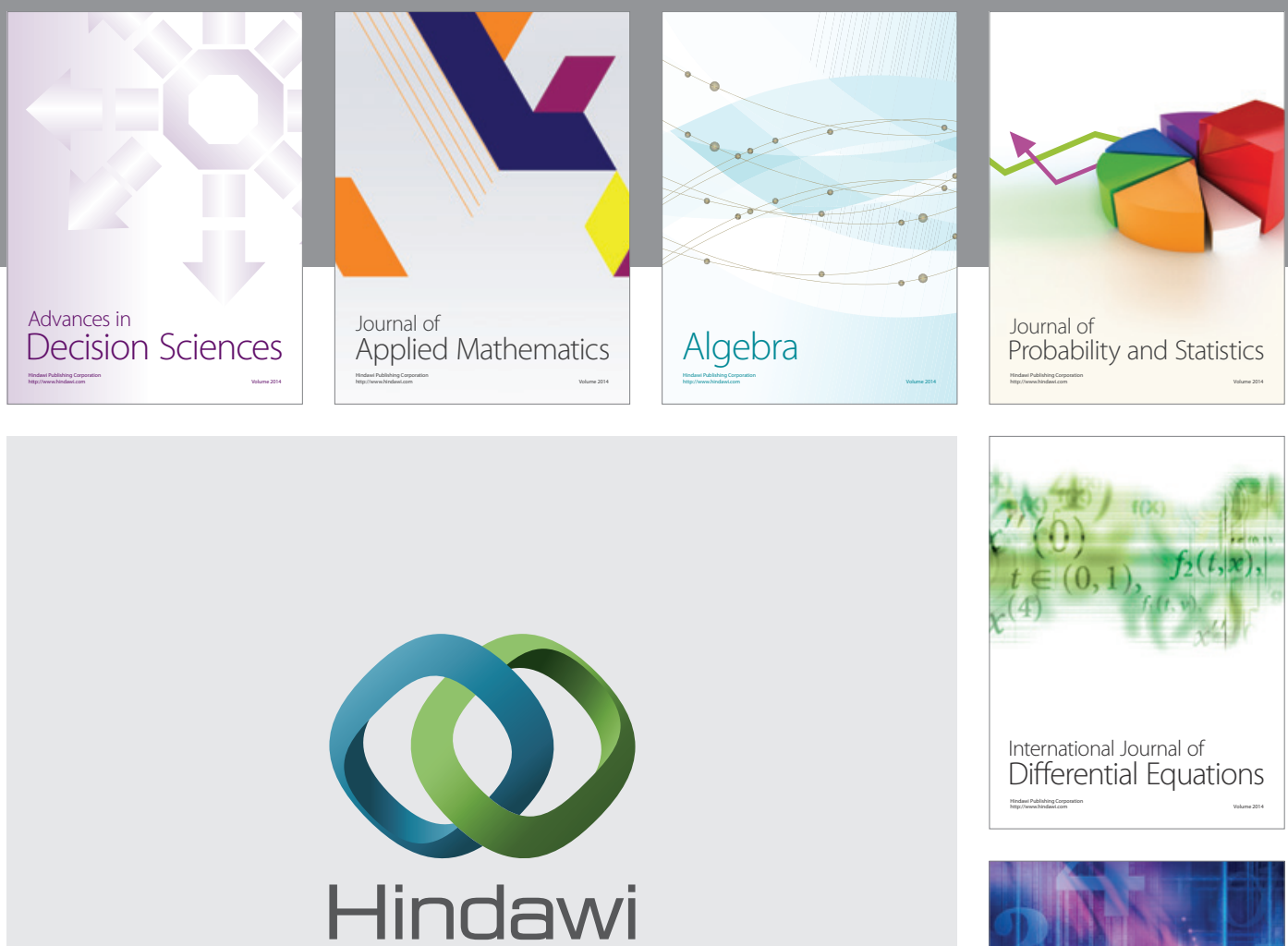

Submit your manuscripts at http://www.hindawi.com
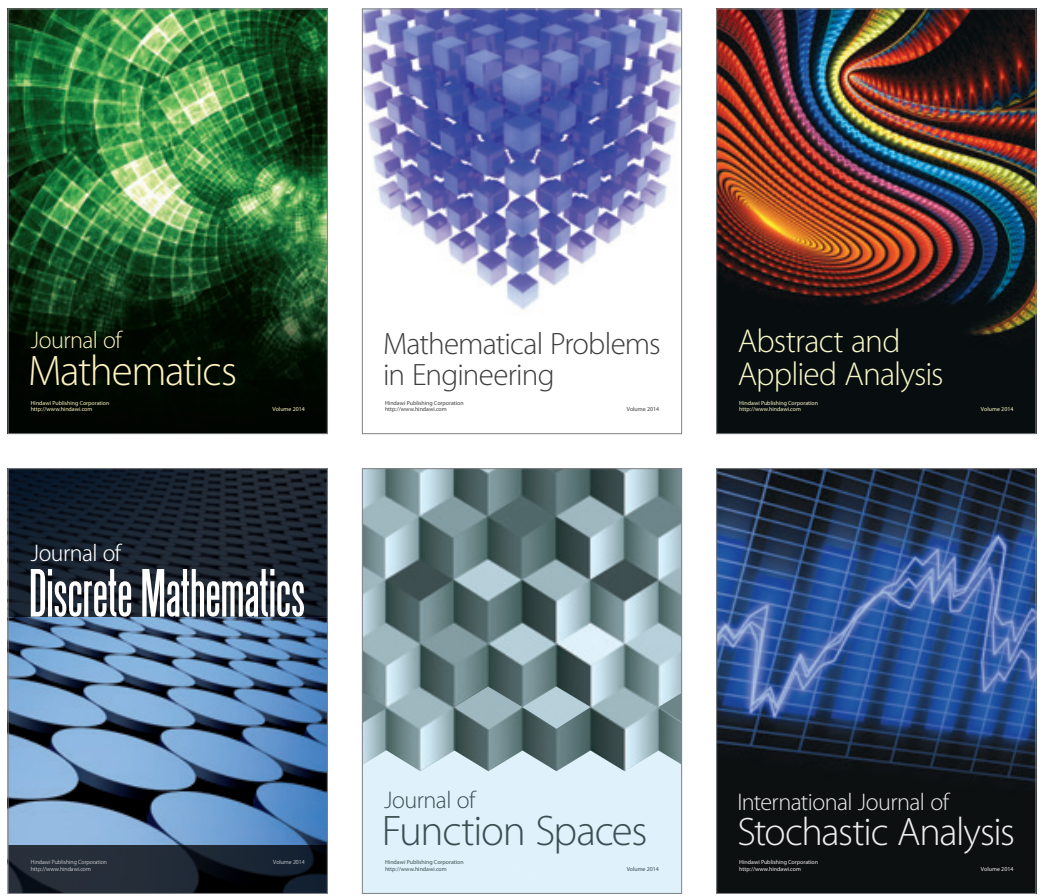

Journal of

Function Spaces

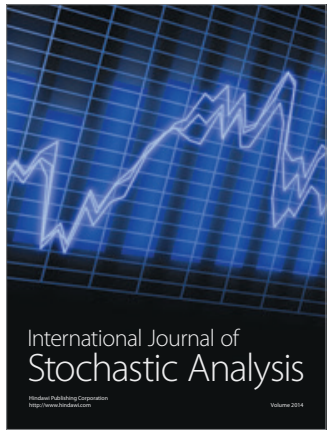

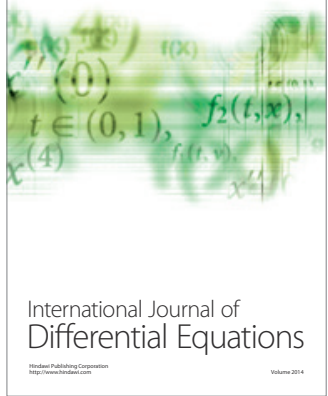
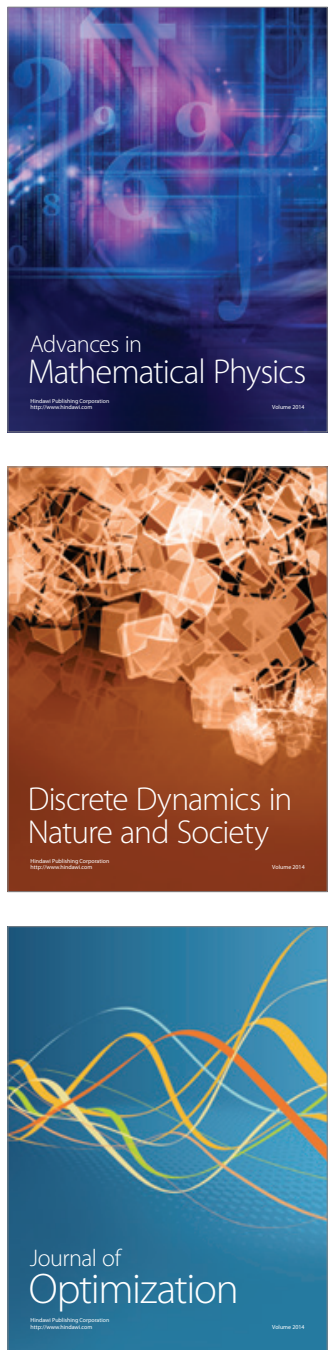